\author{
Shu-hua Ye \\ Shanghai Observatory, Academia Sinica \\ People's Republic of China
}

In recent years, efforts of Astro-Geodynamics research in China have mainly been concerned with the exploration of the regularity and mechanism of the rotational and crustal motion of the Earth as well as with the possible relationships between astronomical factors and earthquakes. In the meantime, our observational devices are under modification and some new techniques have been established. Briefing on these two aspects are given as follows:

\title{
A. PROGRESS OF RESEARCH
}

1. Relations between earthquakes and astronomical phenomena.

Since the disastrous earthquake took place in Xin-Tai (March 1966, magnitude 7), efforts of investigation on the characteristics and mechanism of earthquakes have been carried out on many fronts of disciplines. Everyone tries his best to contribute, even a little, to the possible warning and forecasting of earthquakes, although it is not an easy matter. Work in the astronomical area is :

\subsection{Polar motion}

In the early $1970^{\prime} \mathrm{s}$, the Beijing Observatory investigated the Earth's strain field due to polar motion. They searched for the correlation between abrupt changes of Chandler motion and the occurence of earthquakes, and concluded that, variation of polar motion may be a trigger for big earthquakes. Last year the Shanghai Observatory explored the excitation mechanism of Chandler motion. They found the material displacement field of a big earthquake may excite the Chandler motion, but the energy produced is one order of magnitude less than that needed to maintain this motion (1). Besides, variation of the energy of the Chandler motion, or its amplitude, since 1900, is correlated to the number of earthquake occurences in each year, but no significant correlation has been found between the energy released by global earthquakes and that of the Chandler motion (1). Recently, calculations of the direction and scale of polar motion changes due to 
the displacement field of some very big earthquakes (magnitude 8) have also been done. For example, the Chile earthquake of 1960 might have caused change of polar motion amounting to 0!04 (2).

\subsection{Variation of the Earth's rotation}

The Beijing Observatory examined the frequency of earthquake which has occurred in different regions of China. They found that it correlated with the accelerating and decelerating intervals of the irregular changing of the Earth's rotation. In cooperation with the Department of Geography of the Shanghai Normal University, the Shanghai Observatory found that significant correlations existed between seasonal variation of the Earth's rotation and earthquakes (greater than magnitude 6) occurred in different tectonic regions in north and south-western China (3).

\subsection{Variation of the local vertical}

The Beijing Observatory investigated the astronomical time and latitude observations of Beijing, Tienjin, Tokyo, Mizusawa and Belgrade for the years 1965-1976, and ten earthquakes near to the sites mentioned above, that is, within $300 \mathrm{kms}$ for quakes greated than magnitude 7 and within $100 \mathrm{kms}$ for those greater than magnitude 6 . They showed that, 3-5 months before the earthquakes took place, there were some particular variations in the time and latitude observations. They thought that changes of local vertical due to a displacement of underground water would probably be the cause of such variations (4).

The Shanghai Observatory also found variation in the time observations made at the Purple Mountain Observatory before the Liyang earthquake in 1977, with the same characteristics as those indicated by the Beijing Observatory.

\section{1. Earth tides}

The Shanghai Observatory showed that weak correlation existed between Earth tides and earthquakes in China. Effects of the vertical component and the fortnight term seem significant. The Beijing Observatory, Tienjin Latitude Station and the Seismological Bureau of Tienjin investigated possible trigger action of earthquake by Earth tide for different tectonic faults in northern China, as well as the distribution of earthquake according to different phases of the Moon (5).

\subsection{Other astronomical phenomena}

The Centre for Analysis and Prediction of the State Seismological Bureau, studied the energy released by earthquakes for each year, the occurences of meteors, comets, relative position of planets, variation of the Earth's rotation, number of sunspots and the energy of the 
cosmic rays, etc. They found that during the sixteenth and the seventeenth centuries, energy of earthquakes, cosmic rays, meteors occurence and the variation of Earth's rotation, all attained their peak values, and also for the year 1976.

\section{Polar motion}

\subsection{Secular motion}

There are some conflicts in the traces of secular polar motion given by different authors because of the poor precision of observations. In 1977, several Chinese observatories cooperated with the Department of Astronomy of the Nanjing University, investigated the secular motion by using all the classical observations available, and established the JYD polar coordinates system which referred to the mean pole of 1968. Down from 1962, the secular trace of the BIH system was mainly on the $x$ direction, and that of the IPMS system was mainly on the $y$ direction, while that of our JYD system was situated somewhere between then. In recent years, the Chinese results are more close to that of the BIH (6). The Shanghai Observatory examined the new values of the ILS for 80 years, a secular motion of 0.003 per year along $63^{\circ} \mathrm{W}$ has been found. Besides, there seems to exist a libration of about 30 years. A local latitude variation of the UKiah station amounted to 0!' 003 per year has also been detected (7).

\subsection{Long-periodic terms}

The Shanghai Observatory analyzed the polar motion of 1900-1968 by means of auto-regression method and obtained long-periodic terms of $40,30,18.6$ and 13 years. They found that the power spectrum of the $x$ and $y$ components was closely similar to the spectrum of the $x$ and $y$ components of the atmospheric vertical stress torque respectively, which implied that atmospheric fluctuation may be one of the sources of the long-periodic polar motion (8).

\subsection{Chandler term}

The Shaanxi Observatory analyzed the frequency spectrum of the Chandler term for 1900-1969 by FFT and periodogram method. They thought that the multi-peak results found probably come from some external modulation with unknown origins, and solar activity may be one of the external disturbances (9) (10).

The Shanghai Observatory, after comparing various models of modulation, concluded that, as the forced polar motion mainly comes from quasi-annual meteorological excitation, and because the forced motion has been represented by annual term only, the complexity of the actual forced motion would be combined in the Chandler motion, and the multi-peak characteristic follows (11). 
The Purple Mountain Observatory discussed the relation between amplitude and frequancy variation of the Chandler motion (12). A11 these observatories found the period of Chandler motion around 1.19 year.

The Beijing Observatory suggested an Earth model with a thin separate layer between the outer part and the intter body, and discussed the characteristics of the Chandler motion (13).

Department of Astronomy of the Nanjing University analyzed the free wobble of the pole by means of Poincare's Earth model with a liquid core and they showed that, under the effect of the liquid core, the coplaner relationship between the instantaneaous axis, the axis of figure and the axis of angular momentum of the Earth would no longer hold.

\section{Variation of the Earth's rotation}

In cooperation with the Department of Mathematics of Fu-Dan University, the Shanghai Observatory investigated the variations of the rotation of the Earth by means of periodogram method and they found that for the years 1820-1970, changes of the Earth's rotation would be well fitted by combination of 11 periodic terms ranging from 9 to 89 years and a 179 years long-term variation (14). Analysis by AR method showed similar periodic terms which also fairly coincided with the frequency spectrum of the $y$ component of polar motion (15).

The Beijing Observatory studied 83 central eclipses recorded in Chinese historical literature, dating from 2700 years ago through 14 centuries and they obtained the secular deceleration for this period as $1.73 \times 10^{-10}$ per year (16). The Beijing Observatory and the Institute for the History of Natural Science discussed the variation of the Earth's rotation in 134 B.C. - 1629 A.D. using 69 timing records of solar eclipses in China. The Shaanxi Observatory is also studying the records of ancient solar eclipses and is trying to separate more precisely the variation of Earth rotation with the acceleration of the Moon.

\section{Local variation}

Local terms in latitude for all observational series have been investigated by the Beijing, Shanghai, Shaanxi Observatories as we11 as by the Department of Astronomy of the Nanjing University. The Shanghai Observatory studied 15 series of European latitude observations and 4 nonpolar periodic terms of 2 to 6 years have been found. The phases of these terms were correlated to the longitude of the observing sites and possible $E-W$ and $W-E$ regional motions were suspected (17). For the time observation similar periodic terms were also detected.

The Shaanxi Observatory found that the time and latitude local 
terms of Chinese observatories as compared with the BIH system were concentrated in phases (mainly in the same quadrant). Error of the solar semi-annual term and a common local term were detected. After allowing for these two terms, the actual local terms of each observatory were much reduced and more stable (18).

5. Earth tides and nearly diurnal nutation

The Institute of Geodesy and Geophysics (in Wuhan) discussed the effects of Earth tides on the time and latitude, and deduced the Love numbers from Chinese observations (19).

The Shanghai Observatory analyzed the time observations for 19731975 of 6 instruments in China by means of maximum entropy method and the $\mathrm{M}_{2}, \mathrm{O}_{1}, \mathrm{M}_{1}, \mathrm{M}_{\mathrm{f}}$ waves were detected; there were also waves ranging from 33 to $11 i$ days and $k=0.266$ was deduced (20). From the 19 years observations of a Danjon astrolabe at the Shanghai Observatory, effects of nearly diurnal nutation and Earth tides were found after reduction of oceanic tides (21) (22).

6. Secular variation in longitude.

The Shanghai Observatory investigated the longitude variation between Eurasia and America continents, the certainty was still poor because of low precision of the observational data (23).

\section{B. MODIFICATION OF OBSERVING DEVICES AND ESTABLISHMENT OF SOME NEW TECHNIQUES}

\section{Classical instruments}

The Nanjing Astronomical' Instruments Factory in cooperation with the Shanghai, Beijing, Shaanxi observatories, designed and manufactured Chinese photoelectric astrolabes (Type I and II (24), and one PZT. The high quality of these instruments has been shown by their observational results, which are comparable to those of the first-grade instruments of the world.

Modifications of the photoelectric transits are being done. The Shanghai Observatory has finished one semiautomatic photoelectric transit with remote controlled TV guiding. The Purple Mountain Observatory just finished an automatic transit with a photoelectric tracking system and remote control by an electronic computer. A11 the Chinese transit instruments have been upgrading their level reading devices.

\section{Doppler tracking of artificial satellites}

By using a Chinese made Doppler receiver, the Shanghai observatory established forecasting, observing and processing work 
for Doppler tracking, and the station coordinates accurate in $\pm 4 \mathrm{~m}$ have been obtained by means of the broadcasting ephemeris of the NNSS satellites. Doppler tracking receivers have been set up at all the Chinese observatories. Two CMA-722B receivers participated in the MERIT short campaign. The one located at the Shanghai Observatory was operated jointly by the Research Institute of the National Surveying and Mapping Bureau and the Shanghai Observatory; the other one located at the Purple Mountain Observatory was operated jointly by the Wuhan College of Geodesy and Cartography and the Purple Mountain Observatory.

\section{Laser ranging of artificial satellite}

The Shanghai, Beijing and Yunnan observatories established their laser ranging systems, consisting of ruby lasers with pulse width of 25 ns (25) (26). In cooperation with the Shanghai Institute of Optics and Fine Mechanics, the Shanghai Observatory has upgraded the laser ranging system, using a Nd/YAG laser of $4 \mathrm{~ns}$ pulse width, and more advanced electronic system. This system participated in the MERIT short campaign, for GEOS-3 tracking, a new and better ranging system capable for LAGEOS tracking is expected to be used in the main campaign.

4. VLBI

The Shanghai Observatory has established an experimental system VLBI with a pair of 6-meter antennas, H-masers and narrow band recorders operating on $21 \mathrm{~cm}$ for very strong radio sources. A new 6meter antenna is just setting up, and better electronic system and a MARK II terminal are under consideration.

As we have seen, research activities in China on Astrogeodynamics are on the way of developing. Astro-geodynamical research needs precise observational data. Upgrading our instruments and techniques is still the key problem in the coming 5 to 10 years. At present, observational and theoretical researchers are facing the challenge of effective detection and discussion of the very high spatial and temporal resolutions of the rotational and crustal motion of the Earth and their relations to the external and internal gravitational and electromagnetic fields. The MERIT campaign now under organisation would undoubtedly promote our efforts in observational techniques and theoretical discussions. 


\section{REFERENCES}

(1) Zhao Ming, Song Guoxuan, 1981, Chandler Wobble and Earthquakes, Kexue Tongbao, vol. 26, $\mathrm{N}^{\circ} 3,253-256$.

(2) Song Guoxuan et a1., 1980, On the contribution of the displacement field induced by earthquake to the Chandler motion, Proc. Chinese Symp. on Astrometry at Shanghai.

(3) Yu Chihying, Lo Shihfang et al., 1974, A preliminary analysis of the relation between the seasonal variation of the Earth's rotation and the earthquake, Acta Geophysica Sinica, vol. 17, N ${ }^{\circ} 1,44-50$.

(4) Li Zhisen, Zhang Guodong et a1., 1978, Correlation between the short anomalies of residuals of astronomical time and latitude and the major earthquakes around the observatories, Acta Geophysica Sinica, vol. $21, \mathrm{~N}^{\circ} 4,278-291$.

(5) Li Guoqing et a1., 1980, On the Earth's tide and earthquakes in North China, Proc. Chinese Symp. on Astrometry at Shanghai.

(6) Polar Motion Collaboration Group, 1979, Research on the problem of the pole coordinations in the Years 1949-1976, Acta Astronomical Sinica, vol. 20, $\mathrm{N}^{\circ} 1,1-8$.

(7) Zhao Ming, Zheng Dawei, A new sequence of ILS pole coordinates and secular polar motion, Acta Astronomical Sinica, vol. $22, \mathrm{~N}^{\circ} 1,18-27$.

(8) Zhao Ming, Zheng Dawei, 1980, On the discussion of secular polar motion, Acta Astronomical Sinica, vol. 21, $\mathrm{N}^{\circ} 1,69$.

(9) Wu Shouxian, Wang Shuhe, Hua Yingmin, 1979, Characteristics of Chandler's polar wobble, Acta Astronomical Sinica, vo1. 20, $\mathrm{N}^{\circ} 2$, 126-133.

(10) Wu Shouxian, Wang Shuhe, Hua Yingmin, 1980, Modulation phenomenon on the Chandler's polar motion, Proc. Chinese Symp. on Astrometry at Shanghai.

(11) Zhao Ming, 1979, On the free wobble of the Earth's pole, Annals of Shanghai Observatory Academia Sinica, $\mathrm{N}^{\circ} 1,1-14$.

(12) Yang Benyou, Shi Guang-cheng, 1979, Main characteristics of annular motion and free wobble of Earth pole, Kexue Tongbao, $\mathrm{N}^{\circ} 14,646-649$.

(13) Chang Huanzhi, 1980, On Chandler wobble, Proc. Chinese Symp. on Astrometry at Shanghai.

(14) Luo Shifang et a1., 1974, Analysis of the periodicity of the irregular rotation of the Earth, Acta Astronomical Sinica, vol. 15, $\mathrm{N}^{\circ} 1,79-85$.

(15) Zheng Dawei, Zhao Ming, 1979, Application of autoregressive technique to Astronomy and Geodynomics, Acta Astronomical Sinica, vol. 20, $\mathrm{N}^{\circ} 3,301-307$.

(16) Li zhisen et al., 1980, The change of the Earth's rotation from the ancient central eclipses of China, Proc. Chinese Symp. on Astrometry at Shanghai.

(17) Li Zhengxin, 1981, Non-polar nature of some Zong periodic terms of astronomical latitude in Europe, Kehue Tongbao, vol. 26, $\mathrm{N}^{\circ} 4$, 330-333.

(18) Wang Zhengming et al., 1979, On the analysis of the annual Z-term, Acta Astronomica Sinica, vo1. 20, $\mathrm{N}^{\circ} 1,9-16$. 
(19) Han Tianqui et a1., 1980, Effect of the Moon tides on Latitude observations at Shanghai, Beijing, Wuchang, Proc. Chinese Symp. on Astrometry at Shanghai.

(20) Zheng Dawei, 1978, An analysis of the short-period terms in the Universal Time, Acta Astronomical Sinica, vol. 19, $\mathrm{N}^{\circ} 1,103-108$.

(21) Yang Zhigen, 1980, The analysis of nearly diurnal free wobble using the data observed with a Danjon astrolabe at Shanghai Observatory, Proc. Chinese Symp. on Astrometry at Shanghai.

(22) Yang Zhigen, 1980, Tidal effect observed with the Danjon astrolabe at Shanghai Observatory, Proc. Chinese Symp. on Astrometry at Shanghai.

(23) Gu Zhennian et al., 1979, Relation between the secular variation of Zongitude and the plate motion, Annals of Shanghai Observatory, $\mathrm{N}^{\circ} 1,26-33$.

(24) The Astrolabe Division, Shanghai Observatory, Academia Sinica, 1976, An analysis of the data derived from the first year observations with the photoelectric astrolabe type II, Acta Astronomical Sinica, vol. 17, $\mathrm{N}^{\circ} 1,27-33$.

(25) Yang Fumin et a1., 1979, The satelzite Zaser ranging system at Shanghai Observatory, Annals of Shanghai Observatory Academia Sinica, $\mathrm{N}^{\circ} 1,83-87$.

(26) Huang Songnian et al., 1979, A preliminary analysis of the accuracy of the satelitte zaser ranging system at Shanghai Observatory, Annals of Shanghai Observatory Academia Sinica, $N^{\circ} 1,88-91$. 